\title{
To have neighbour's fare: extending the molecular toolbox for Streptococcus pneumoniae
}

Correspondence
Oscar P. Kuipers
o.p.kuipers@rug.nl

Received 14 September 2005

Revised 8 November 2005

Accepted 8 November 2005

\author{
Tomas G. Kloosterman, Jetta J. E. Bijlsma, Jan Kok and Oscar P. Kuipers
}

Department of Molecular Genetics, University of Groningen, Groningen Biomolecular Sciences and Biotechnology Institute, PO Box 14, 9750 AA Haren, the Netherlands

In past years, several useful genetic tools have been developed to study the molecular biology of Streptococcus pneumoniae. In order to extend the existing spectrum of tools, advantage was taken of the toolbox originally developed for the closely related bacterium Lactococcus lactis, which was adapted for the manipulation of $S$. pneumoniae. The modified tools are as follows. (i) An improved nisin-inducible (over)expression system (NICE). The nisRK genes, encoding a two-component system essential for transcriptional activation in response to nisin, were integrated into the bgaA locus of $S$. pneumoniae D39. In this strain, D39nisRK, addition of nisin resulted in the overexpression of several genes placed under the control of the nisin-inducible promoter, while no detectable expression was observed in the absence of nisin. (ii) A lacZ reporter system. Using strain D39nisRK, which lacks endogenous $\beta$-galactosidase activity, the usefulness of the lacZ reporter vector $\mathrm{pOR} 13$ for the generation of chromosomal transcriptional fusions was demonstrated. In addition, the repA gene, necessary for the replication of pORI13, was introduced into the $b g a A$ locus, thereby generating a background for plasmid-based promoter expression studies. (iii) A simplified chemically defined medium, which supports growth of all sequenced S. pneumoniae strains to a level comparable to that in complex medium. (iv) A system for the introduction of unmarked deletions and mutations into the chromosome, which is independent of the genotype of the target strain. Most of these systems were successfully applied in strains R6 and TIGR4 as well. In addition, the tools offer several improvements and advantages compared to existing ones. Thus, the molecular toolbox for S. pneumoniae has been successfully extended.

\section{INTRODUCTION}

Streptococcus pneumoniae is a human pathogen, and causes meningitis, pneumonia, otitis media and bacteraemia, especially in children and the elderly. Treatment and prevention of infection is primarily based on antibiotics and vaccines (Bogaert et al., 2004). Current vaccines are directed against the polysaccharide capsule, but they do not cover the entire spectrum of different serotypes, and may offer only limited protection in children younger than 2 years (Bogaert et al., 2004). Furthermore, multi-antibiotic-resistant strains of $S$. pneumoniae have emerged in recent years, and are now a serious problem (Hancock, 2005). To be able to combat infections caused by $S$. pneumoniae in the future, new vaccines and antimicrobials are required. Therefore, a major effort is currently being made to dissect the molecular mechanisms underlying infection by $S$. pneumoniae.

\footnotetext{
Abbreviations: CDM, chemically defined medium; GFP, green fluorescent protein; NICE, nisin controlled expression.

A description of the chemically defined medium together with sequence data for plasmids pTK3 and PTK5 is available as supplementary data with the online version of this paper.
}

Various molecular tools have been described to study the molecular biology of $S$. pneumoniae, such as inducible promoters, lac $Z$ and green fluorescent protein (GFP) reporter vectors, and methods to construct mutant strains (Acebo et al., 2000; Chan et al., 2003; Claverys et al., 1995; Eichenbaum et al., 1998; Lee et al., 1998; Sung et al., 2001).

Here, we report the development and use of several additional molecular tools for S. pneumoniae, which were adapted from existing tools for the related bacterium Lactococcus lactis. These include an improved nisin-controlled gene expression system (NICE), a chemically defined medium (CDM), a plasmid-based lacZ reporter system, and a method to generate unmarked mutations in the chromosome. These tools extend the existing toolkit for the basic genetic manipulation of $S$. pneumoniae, and they are applicable in the sequenced strains R6 (D39) and TIGR4, and, most likely, in other strains as well.

\section{METHODS}

Strains and growth conditions. Strains used in this study are listed in Table 1 and were stored in $10 \%(\mathrm{v} / \mathrm{v})$ glycerol at $-80^{\circ} \mathrm{C}$. S. pneumoniae was routinely grown as standing cultures in M17 broth 
Table 1. Strains and plasmids used in this study

$\mathrm{Amp}^{\mathrm{R}}$, ampicillin resistance; $\mathrm{Cm}^{\mathrm{R}}$, chloramphenicol resistance; $\mathrm{Em}^{\mathrm{R}}$, erythromycin resistance; $\mathrm{Km}^{\mathrm{R}}$, kanamycin resistance; Spec ${ }^{\mathrm{R}}$, spectinomycin resistance; $\operatorname{Trmp}^{\mathrm{R}}$, trimethoprim resistance.

\begin{tabular}{|c|c|c|}
\hline Strain/plasmid & Description & Reference or source \\
\hline \multicolumn{3}{|l|}{ S. pneumoniae } \\
\hline D39 & Serotype 2 strain, cps 2 & Avery et al. (1944) \\
\hline TIGR4 & Serotype 4 strain & Tettelin et al. (2001) \\
\hline R6 & D39 (cps2 2538-9862) with increased transformation efficiency & Hoskins et al. (2001) \\
\hline D39nisRK & D39 $\Delta$ bgaA:: nisRK; $\operatorname{Trmp}^{\mathrm{R}}$ & This work \\
\hline D39repA & D39 $\Delta$ bgaA:: repA; $\operatorname{Trmp}^{\mathrm{R}}$ & This work \\
\hline TIGR4nisRK & TIGR4 $\Delta b g a A::$ nisRK; $\operatorname{Trmp}^{\mathrm{R}}$ & This work \\
\hline TIGR4repA & TIGR4 $\Delta b g a A:: r e p A ; \operatorname{Trmp}^{\mathrm{R}}$ & This work \\
\hline R6nisRK & R6 $\triangle b g a A:: n i s R K ; \operatorname{Trmp}^{\mathrm{R}}$ & This work \\
\hline R6repA & R6 $\Delta b g a A:: r e p A ; \operatorname{Trmp}^{\mathrm{R}}$ & This work \\
\hline TK100 & D39nisRK $\Delta g \ln A ; \mathrm{Spec}^{\mathrm{R}}$ & Laboratory collection \\
\hline TK101 & D39 $\Delta g \ln R$, containing unmarked chromosomal deletion of $g \ln R$ & This work \\
\hline \multicolumn{3}{|c|}{ 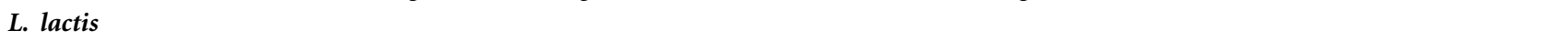 } \\
\hline NZ9000 & MG1363 $\Delta p e p N::$ nisRK & Kuipers et al. (1998) \\
\hline LL108 & MG1363 RepA $^{+}$(multi-copy); $\mathrm{Cm}^{\mathrm{R}}$ & Leenhouts et al. (1998a) \\
\hline \multicolumn{3}{|l|}{ E. coli } \\
\hline EC1000 & $\begin{array}{l}\mathrm{Km}^{\mathrm{R}} \text {; MC1000 derivative carrying a single copy of the pWV01 } \\
\text { repA gene in } \operatorname{glg} B\end{array}$ & Leenhouts et al. (1996) \\
\hline \multicolumn{3}{|c|}{ 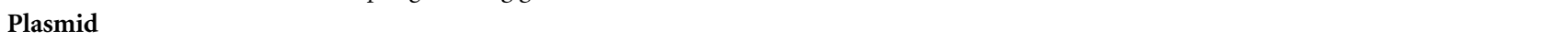 } \\
\hline pORI13 & $\mathrm{Em}^{\mathrm{R}} ;$ ori $^{+}$rep $A^{-} ;$promoterless lacZ & Sanders et al. (1998) \\
\hline pORI280 & $\begin{array}{l}\mathrm{Em}^{\mathrm{R}} \text {; } \text { ori }^{+} \text {rep }^{-} \text {; deletion derivative of } \mathrm{pWV} 01 \text {; constitutive lacZ } \\
\text { expression from P32 promoter }\end{array}$ & Leenhouts et al. (1996) \\
\hline pORI28 & $\mathrm{Em}^{\mathrm{R}} ;$ ori $^{+} r e p A^{-} ;$deletion derivative of $\mathrm{pWV} 01$ & Leenhouts et al. (1996) \\
\hline pNZ8048 & $\mathrm{Cm}^{\mathrm{R}}$; nisin-inducible PnisA & de Ruyter et al. (1996b) \\
\hline pNG8048E & $\mathrm{Cm}^{\mathrm{R}} \mathrm{Em}^{\mathrm{R}}$; nisin-inducible PnisA, pNZ8048 derivative & Laboratory collection \\
\hline pKOT & $\begin{array}{l}\text { Amp }^{\mathrm{R}} \operatorname{Trmp}^{\mathrm{R}} \text {; pBluescript II KS + containing trmp gene under } \\
\text { constitutive P32 promoter }\end{array}$ & $\begin{array}{l}\text { Laboratory collection; } \\
\text { P. W. Hermans* }\end{array}$ \\
\hline pUC23rep3 & $\mathrm{Amp}^{\mathrm{R}}$; carrying the pWVOI repA gene under control of promoter P23 & Leenhouts et al. (1991) \\
\hline pJB1 & $\mathrm{Cm}^{\mathrm{R}}$; pNG8048E carrying spr0567-myc behind nisA promoter & This work \\
\hline pJB2 & $\mathrm{Em}^{\mathrm{R}} ;$ pORI13 $:$ :P $\operatorname{cps} A$ & This work \\
\hline pTK1 & $\mathrm{Em}^{\mathrm{R}} \operatorname{Trmp}^{\mathrm{R}}$; pORI28 containing P32:: trmp gene from pKOT & This work \\
\hline pTK2 & $\mathrm{Em}^{\mathrm{R}} \operatorname{Trmp}^{\mathrm{R}}$; pTK1 containing left and right parts of D39 bgaA & This work \\
\hline pTK3 & $\mathrm{Em}^{\mathrm{R}} \operatorname{Trmp}^{\mathrm{R}} ; \mathrm{pTK} 2$ containing NZ9700 nisRK genes & This work \\
\hline pTK4 & $\begin{array}{l}\mathrm{Em}^{\mathrm{R}} \operatorname{Trmp}^{\mathrm{R}} ; \text { pTK1 containing left and right flanking regions from } \\
\mathrm{D} 39 \text { bgaA }\end{array}$ & This work \\
\hline pTK5 & $\mathrm{Em}^{\mathrm{R}} \operatorname{Trmp}^{\mathrm{R}}$; pTK4 containing repA from pUC23rep3 & This work \\
\hline pTK6 & $\mathrm{Cm}^{\mathrm{R}}$; pNG8048E carrying $g \ln A$ behind nis $A$ promoter & This work \\
\hline pTK7 & $\mathrm{Em}^{\mathrm{R}} ;$ pORI280 carrying $g \ln R$ deletion & This work \\
\hline
\end{tabular}

*University Medical Center St Radboud, The Netherlands.

(Terzaghi \& Sandine, 1975) containing $0 \cdot 25 \%(w / v)$ glucose (GM17) or in Todd-Hewitt broth supplemented with $0.5 \%$ yeast extract (THY) at $37^{\circ} \mathrm{C}$. For growth on plates, $1-5 \%(\mathrm{v} / \mathrm{v})$ defibrinated sheep blood (Johnny Rottier, Kloosterzande, The Netherlands) was added to GM17 agar. L. lactis was grown as standing cultures in M17 supplemented with $0.5 \%(\mathrm{w} / \mathrm{v})$ glucose at $30^{\circ} \mathrm{C}$. Escherichia coli EC1000 was grown in TY broth in a shaking incubator at $37^{\circ} \mathrm{C}$. Chloramphenicol and erythromycin were used at concentrations of 2.5 and $0.25 \mu \mathrm{g} \mathrm{ml}^{-1}$ for S. pneumoniae, and 5 and $4 \mu \mathrm{g} \mathrm{ml}^{-1}$ for L. lactis, respectively. Erythromycin was used at a concentration of $120 \mu \mathrm{g} \mathrm{ml}^{-1}$ for E. coli. Trimethoprim was used at a concentration of $15 \mu \mathrm{g} \mathrm{ml}^{-1}$ for S. pneumoniae. When appropriate, $0.006 \%(\mathrm{w} / \mathrm{v})$
$\mathrm{X}$-Gal was used in plates. A nisin stock was obtained by extracting nisaplin (Aplin and Barrett, Danisco, Denmark) $1: 1$ (w/v) with 50\% ethanol, spinning the suspension for $1 \mathrm{~min}$ at $20000 \mathrm{~g}$ in a table-top centrifuge and discarding the pellet. The supernatant contained approximately $20 \mu \mathrm{g}$ nisin $\mathrm{ml}^{-1}$ and was stored at $-20{ }^{\circ} \mathrm{C}$. For induction of the nisA promoter, nisin was used at the concentrations indicated in the Results. Working stock solutions with the appropriate nisin concentration were freshly prepared in $50 \%$ ethanol before each experiment.

DNA isolation and manipulation. Chromosomal DNA was isolated from $L$. lactis and S. pneumoniae according to the method of 
Johansen \& Kibenich (1992). Plasmid DNA was isolated using the plasmid isolation kit from Roche. DNA manipulation was done according to standard procedures. L. lactis and E. coli were transformed with plasmid DNA by electroporation (Holo \& Nes, 1995). S. pneumoniae was transformed by growing the strain at $37^{\circ} \mathrm{C}$ in GM17 to an $\mathrm{OD}_{600}$ of $0 \cdot 1$. The competence-stimulating peptide CSP-1 was used at $100 \mathrm{ng} \mathrm{ml}^{-1}$ to induce competence (Pozzi et al., 1996). Transformants were selected on GM17 agar with the appropriate antibiotic. Southern blotting was done as described by Chomczynski \& Qasba (1984). Probe labelling, hybridization and detection were performed using the ECL direct nucleic acid labelling system according to the specifications of the manufacturer (Amersham Pharmacia Biotech).

Plasmid and strain construction. Primers used in this study are listed in Table 2. The nisRK genes were introduced into the bgaA gene (spr0565) on the chromosome of $S$. pneumoniae as follows. Primers trpm-Fp and trmp-Rp were used to PCR-amplify the trmpR marker from pKOT. Using E. coli EC1000 as host, the PCR fragment was inserted into the HindIII site of pORI28 in the same orientation as the emR gene, yielding pTK1. The $5^{\prime}$ and $3^{\prime}$ ends of bgaA gene were amplified from D39 chromosomal DNA with primer pairs bgaA-1/bgaA-2 and bgaA-3/bgaA-4, and were cloned into the MluI/BamHI and KpnI/BglII sites of pTK1, respectively, resulting in pTK2. Finally, the nisRK genes, which were amplified from chromosomal DNA of L. lactis NZ9700 with primers NisRKFp-term/NisRK-Rp, were cloned into the PstI/BamHI sites of pTK2, resulting in pTK3. Both pTK2 and pTK3 were generated in L. lactis LL108.

The repA gene was introduced into the $b g a A$ locus in a similar manner using E. coli EC1000 as host. Regions of approximately $800 \mathrm{bp}$ surrounding bgaA were amplified from chromosomal DNA of D39 using primers R6_bgaA-5/6 and R6_bgaA-7/8 and cloned into the $X b a \mathrm{I} / B a m \mathrm{HI}$ and $K p n \mathrm{I} / B g l \mathrm{II}$ sites of pTK1, resulting in pTK4. Subsequently, the repA gene, amplified from plasmid pUC23rep3 with primers RepA-Fprimer_term/RepA-Rprimer, was cloned into the $\mathrm{XbaI} /$ PstI sites of pTK4, giving pTK5.

S. pneumoniae $\mathrm{D} 39$ nisRK, harbouring the nisRK genes integrated via double cross-over in the bgaA locus, was constructed by transformation with a PCR product obtained with $\mathrm{pTK} 3$ as template using primers bgaA-1/bgaA-4 and selecting for trimethoprim-resistant clones. By analogy, a gene cassette was obtained from pTK5 by PCR with primers R6_bgaA-6/R6_bgaA-8, which was used to construct $S$. pneumoniae D39repA. The correctness of the mutations was verified by PCR and Southern blotting. In the same way, strains TIGR4nisRK, TIGR4repA, R6nisRK and R6repA were constructed.

Table 2. Oligonucleotide primers used in this study

Restriction enzyme sites underlined; stem-loop structure (predicted $\Delta G^{0}-20 \cdot 8 \mathrm{kcal} \mathrm{mole}{ }^{-1},-87 \cdot 0 \mathrm{~kJ}$ $\mathrm{mol}^{-1}$ ) in bold.

\begin{tabular}{|c|c|c|}
\hline Primer & Nucleotide sequence $\left(5^{\prime}\right.$ to $\left.3^{\prime}\right)$ & Restriction site \\
\hline trmp-Fp & CCCCAAGCTTGGATTTTTGTGAGCTTGGA & HindIII \\
\hline trmp-Rp & CCCCAAGCTTGTTACGACGCGCATAGACGG & HindIII \\
\hline bgaA-1 & GCTCTAGACAAATCGTTGAACGAGGTGG & $X b a \mathrm{I}$ \\
\hline bgaA-2 & CGGGATCCCCTGTCCAGATAAACTGTCC & BamHI \\
\hline bgaA-3 & GGGGTACCCGCTACCGTCGTTCCAAGCG & KpnI \\
\hline bgaA-4 & GAAGATCTGTAATTTGATAGTCTTGACG & BglII \\
\hline nisRK-Fp-term & $\begin{array}{l}\text { CGGGATCCAGGGAAGGGAGGTGAGAGCCTCCCTT- } \\
\text { CCCTAAAAAAGTGTGTATCTCAATCCTTGG }\end{array}$ & BamHI \\
\hline nisRK-Rp & AAACTGCAGAATCCTTAGAGATTAC & Pst $\mathrm{I}$ \\
\hline R6_bgaA-5 & CGGGATCCCCAAGTGGTATACATGGTATGAC & BamHI \\
\hline R6_bgaA-6 & GCTCTAGAGTTGCTACTAAACGTCTTCAAGG & $X b a \mathrm{I}$ \\
\hline R6_bgaA-7 & GGGGTACCGCAGGATTAGTAGTTACTAAAGG & $K p n \mathrm{I}$ \\
\hline R6_bgaA-8 & GAAGATCTGAAAACGACAGAGAAATTCTCC & BglII \\
\hline RepA-Fprimer_term & $\begin{array}{l}\text { CGGGATCCAGGGAAGGGAGGTGAGAGCCTCCCTT- } \\
\text { СССTAAAAAATTCGAGCTCGCCСАTCСТTTCAT }\end{array}$ & BamHI \\
\hline RepA-Rprimer & AAAACTGCAGCCCCTTCGACTTTCGTCAGGGGGC & Pst $\mathrm{I}$ \\
\hline glnA_R6-7 & GGCGGTCTCTCATGCCAATCACAGCTGCAG & BsaI \\
\hline glnA_R6-8 & GCTCTAGATGTGACTGTTTACCCACAG & $X b a \mathrm{I}$ \\
\hline 659 for & CATGCCATGGAAAAATGGCAAACATGTG & $\mathrm{NcoI}$ \\
\hline 659rev & $\begin{array}{l}\text { TGCTCTAGACTAGTTAAGATCTTCTTCTGAAATAA- } \\
\text { GTTTTTGTTCGGCTAATTCCTTCAAAGTTTG }\end{array}$ & $X b a \mathrm{I}$ \\
\hline cpsArev + 50 & AACTGCAGACACTTCGCTTCACTTTCTG & Pst $\mathrm{I}$ \\
\hline cpsAfor-400 & CGGGATCCAAGTACCGCCATAGAGCG & $X m a \mathrm{I}$ \\
\hline glnR_R6-1 & TGCTCTAGAGGTGGTGACTCAGCTGCCGC & $X b a \mathrm{I}$ \\
\hline glnR_R6-2 & CGGGATCCССТTCATTTACAATTTCCTTC & BamHI \\
\hline glnR-R6-3-ncoi & CATGCCATGGGGTCGCGGTTAGGCAACCGC & NcoI \\
\hline glnR_R6-4 & GAAGATCTCCTTCTGCATCAAACAAGG & BglII \\
\hline
\end{tabular}


Plasmids pTK6 and pJB1 containing glnA (spr0444) and spr0576 under the control of the nisin-inducible nis $A$ promoter were constructed by amplifying the respective genes from chromosomal DNA of strain D39 using primer pairs glnA_R6-7/glnA_R6-8 and 659for/659rev, and cloning the resulting amplicons into the $\mathrm{NcoI} / \mathrm{XbaI}$ sites of pNG8048E. The spr0576 gene was fused to a C-terminal Myc-tag. L. lactis NZ9000 was used as the cloning host.

To construct a transcriptional fusion of lac $Z$ to the promoter of $\operatorname{cps} A$, the fragment was PCR-amplified from chromosomal DNA of $S$. pneumoniae D39 with primer pair cpsArev +50 /cpsAfor-400 and cloned into the XmaI/PstI sites of pORI13, yielding pJB2. L. lactis LL108 was used as the cloning host.

pTK7, containing the deleted $g \ln R$ gene (spr0443) surrounded by fragments of approximately $800 \mathrm{bp}$, was constructed by cloning the PCR products amplified from D39 chromosomal DNA with primer pairs glnR_R6-1/glnR_R6-2 and glnR-R6-3-ncoi/glnR_R6-4 in the XbaI/ BamHI and NcoI/BglII sites of pORI280, using L. lactis LL108 as host.

Enzyme assays. Cell-free extracts were made by resuspending cell pellets from $2 \mathrm{ml}$ of culture in $20 \mathrm{mM}$ Tris/ $\mathrm{HCl}(\mathrm{pH} \mathrm{7.5)}$ and disruption of cells by shaking them for $1 \mathrm{~min}$ with glass beads $(75-150 \mu \mathrm{m})$ in a Biospec Mini-BeadBeater-8. Glutamine synthetase activity (transferase reaction) was determined as described by Fisher \& Sonenshein (1984). $\beta$-Galactosidase activity was determined in cell suspensions permeabilized by chloroform and SDS, as described by Israelsen et al. (1995).

Detection of the Spr576-Myc fusion protein by Western blotting. Cells from $1 \mathrm{ml}$ of cell culture grown in GM17 were harvested in $1 \mathrm{ml} \mathrm{PBS}$ and washed twice. Whole-cell samples were diluted in sample buffer (4\% SDS, $2 \%$ 2-mercaptoethanol, $20 \%$, $\mathrm{v} / \mathrm{v}$, glycerol, $125 \mathrm{mM}$ Tris/ $\mathrm{HCl}, \mathrm{pH} 6 \cdot 8,0 \cdot 1 \mathrm{mg}$ bromophenol blue $\mathrm{ml}^{-1}$ ), heated for $5 \mathrm{~min}$ at $100^{\circ} \mathrm{C}$, fractionated by $12.5 \%$ SDSPAGE and transferred to a nitrocellulose membrane. Spr576-Myc was detected with ECL (Amersham Biosciences) on immunoblots after incubation with a 1:5000 dilution of an anti-Myc monoclonal antibody (Gentaur, Brussels, Belgium), followed by a 1:10 000 dilution of anti-mouse IgG peroxidase (Amersham Biosciences).

\section{RESULTS AND DISCUSSION}

\section{Construction of an improved NICE system for overexpression in S. pneumoniae}

NICE is used mainly in L. lactis, but has also been applied successfully in other Gram-positive bacteria (Eichenbaum et al., 1998; Kleerebezem et al., 1997; Mierau \& Kleerebezem, 2005). The core of NICE is formed by the NisRK twocomponent system, which in the presence of nisin activates expression of the nis $A$ and nis $P$ promoters in a dosedependent manner (de Ruyter et al., 1996a; Kuipers et al., 1995). Nisin is a peptide bacteriocin belonging to the class of lantibiotics, and is produced by certain strains of L. lactis. An advantage of induction of expression with nisin is that it is metabolically inert at subinhibitory concentrations, unlike sugars and other metabolically active compounds that have been used in S. pneumoniae (Acebo et al., 2000; Chan et al., 2003).

Two studies describe the application of NICE in S. pneumoniae (Eichenbaum et al., 1998; Luo et al., 2004). In the first study, two plasmids were used, one containing the nisin-inducible promoter, the other the nisRK genes. In this situation, transcription from the nisin-inducible promoter also occurred in the absence of nisin. Furthermore, nisin concentrations just below the growth inhibitory level resulted in only a tenfold overproduction (Eichenbaum et al., 1998). The second study used a single plasmid that contains both the nisin-inducible promoter and the nisRK genes, but in this case, nisin-induced expression was observed only at $30^{\circ} \mathrm{C}$ (Luo et al., 2004).

To circumvent these problems, we have constructed a stable derivative of $S$. pneumoniae D39 (D39nisRK) that harbours nisRK with their native promoter in the bgaA locus (Fig. 1a), thereby abolishing endogenous $\beta$-galactosidase activity (Fig. 3b).

To test whether NICE is functional in strain D39nisRK, the genes encoding glutamine synthetase GlnA and the putatively secreted protein Spr0576 were placed under the control of the nisA promoter in plasmid pNG8048E. In the absence of nisin, there was no detectable expression from the nisA promoter (Fig. 1b, c). Addition of nisin to the medium resulted in expression of $g \ln A$, which was dose-dependent (Fig. 1b). At nisin concentrations of $2-20 \mathrm{ng} \mathrm{ml}^{-1}$, which are far below the MIC value of $500 \mathrm{ng} \mathrm{ml}^{-1}$ (Kramer, 2005), a 20-50-fold higher glutamine synthetase activity was observed compared to the wild-type (Fig. 1b). Nisininduced overexpression of spr0576 resulted in the appearance of two bands on the Western blot (Fig. 1c). The lower band probably represents the protein with the putative signal sequence cut off, and the upper band the unprocessed protein. The appearance of these two bands indicates that nisin induction leads to the production of large quantities of the Myc-tagged Spr0576. Furthermore, we also showed that nisin-induced expression occurs in both the early and the late exponential phase of growth (Fig. 1c).

The nisRK integration cassette could be easily transferred to other strains via double cross-over and selection for resistance to the rarely used antiobiotic trimethoprim. In addition, nisin-dependent expression of GlnA and Spr0576 was equally functional in TIGR4 and R6 derivatives, which carry nisRK integrated in the bgaA locus (data not shown).

Although THY and brain heart infusion (BHI) are often used as growth media for $S$. pneumoniae, we carried out all experiments in GM17 medium, which is a commonly used medium for L. lactis. The growth rate and maximal $\mathrm{OD}_{595}$ of strains D39, R6 and TIGR4 were higher in GM17 than in THY or BHI (data not shown). Also, nisin-induced expression of $g \ln A$ in THY seemed to be lower than that in GM17. As the stability and solubility of nisin are strongly diminished above pH 7 (Rollema et al., 1995), we speculated that this difference was due to the higher $\mathrm{pH}$ of THY, which is $7 \cdot 8$ versus 6.9 for GM17. To test this hypothesis, nisin induction was performed in GM17 medium at various $\mathrm{pH}$ values. In GM17 with a $\mathrm{pH}$ higher than $7 \cdot 5$, the level of induction was strongly decreased, while at lower $\mathrm{pH}$ values, the induction level was comparable to that obtained in standard GM17. 
(a)

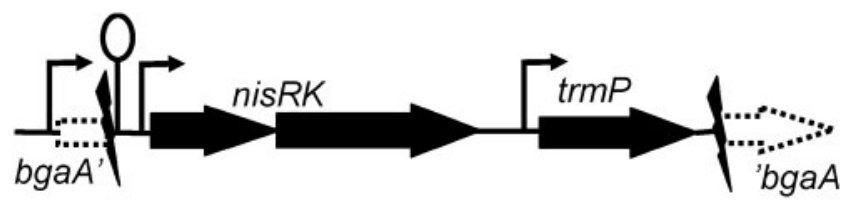

(b)

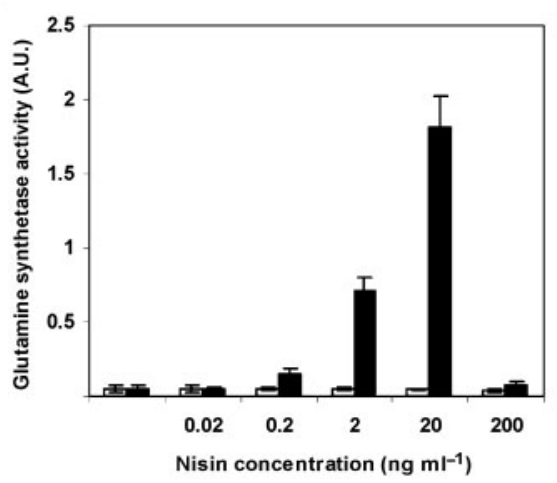

(c)

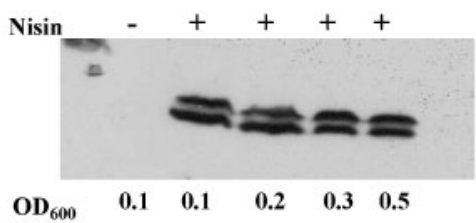

Fig. 1. Characterization of the improved NICE system. (a) Schematic representation of the nisRK genes of L. lactis integrated in the bgaA locus on the chromosome of $S$. pneumoniae. The integration led to the removal of an approximately $3 \mathrm{~kb}$ internal fragment of bgaA. trmP, trimethoprim-resistance marker. Stem-loop indicates terminator structure (predicted $\Delta G^{0}-20 \cdot 8 \mathrm{kcal} \mathrm{mol}^{-1},-87 \cdot 0 \mathrm{~kJ} \mathrm{~mol}^{-1}$ ). Right-pointing arrows represent promoters; lightning symbols represent the site of insertion of the nisRK fragment; the dotted arrow shows the position of the disrupted $b g a A$ gene. (b) Nisin concentrationdependent expression of $g \ln A$ in $S$. pneumoniae D39nisRK, harbouring $g \ln A$ cloned behind the nis $A$ promoter of pNG8048E (pTK6, black bars) or the empty plasmid pNG8048E (white bars). Induction with nisin was done for $1 \mathrm{~h}$ at $\mathrm{OD}_{600} 0.2$ in GM17. (c) Western blot showing nisin-induced expression of the Myc-tagged Spr0576 in D39nisRK harbouring plasmid JB1 at different culture densities $\left(\mathrm{OD}_{600}\right)$ during exponential growth in GM17. The lower band represents Spr0576 with the signal sequence cut off, and the upper band the unprocessed protein. Induction $(+)$ was done for $1 \mathrm{~h}$ with $2 \mathrm{ng}$ nisin $\mathrm{ml}^{-1}$. -, No addition of nisin.

As expected, lowering the $\mathrm{pH}$ of THY to $7 \cdot 5$ or below led to enhanced induction levels (data not shown). Thus, adjustment of the $\mathrm{pH}$ of the growth medium could be a good way to optimize NICE in S. pneumoniae.

\section{Complementation of a gInA deletion mutant with NICE in chemically defined medium}

Previously, complementation in S. pneumoniae has been accomplished with the fucose-inducible promoter ( $\mathrm{Ng}$ et al., 2004). To validate the improved NICE system, we tested whether $g \ln A$, expressed under the control of the nisininducible nisA promoter in S. pneumoniae D39nisRK, could complement a chromosomal deletion in $g \ln A$. In order to do so, we first needed a CDM to ensure that the $g \ln A$ mutant was auxotrophic for glutamine. Although chemically defined media for S. pneumoniae have been described (Adams \& Roe, 1945; van de Rijn \& Kessler, 1980; Willett \& Morse, 1966), they are not commonly used, which could be due to their complexity. We adapted a CDM for L. lactis (Poolman \& Konings, 1988) by supplementing it with pyruvate, adenine, uracil, choline chloride, aspartate and cysteine (supplementary data). This CDM is easy to prepare and supported growth of three S. pneumoniae strains tested, namely R6, D39 and TIGR4, to a level comparable to that in complex media, such as THY and GM17 (data not shown).

The glnA mutant grew well in CDM containing glutamine, but, unlike the wild-type, did not grow in CDM with glutamate instead of glutamine (Fig. 2a). In trans expression of $g \ln A$ from the nisin-inducible promoter restored growth in $\mathrm{CDM}$ with glutamate instead of glutamine to the level of that in CDM containing glutamine (Fig. 2b), demonstrating the suitability of the NICE system we describe here for complementation experiments in S. pneumoniae.

Taken together, we have adapted the NICE system for $S$. pneumoniae by integrating the nisRK genes into the bgaA locus, which allows in trans nisin-inducible expression of, in principle, every gene of $S$. pneumoniae, and solves the limitations observed in earlier studies. In addition, because of the inactivation of endogenous $\beta$-galactosidase activity, the strain can be employed for promoter-expression studies using the E. coli lacZ gene as a reporter, either in combination or not in combination with nisin-controlled expression of the protein of interest.

\section{Plasmid-based lac $Z$ expression system for studying promoter function in $\mathbf{S}$. pneumoniae}

A plasmid-based GFP reporter system has been applied in S. pneumoniae (Bartilson et al., 2001; Marra et al., 2002), but the lac $Z$ reporter systems described to date are based on integrative plasmids (Claverys et al., 1995; Pestova \& Morrison, 1998). The efficiency of integration into the chromosome via insertion-duplication, however, strongly depends on the length of the targeting fragment, which could give problems when a small insert is required (Lee et al., 1998). In addition, an integration plasmid is less suitable for the detailed dissection of promoter function, for example by point mutations or deletions.

We developed a plasmid-based lacZ reporter system using the lactococcal pWV01 replicon (Leenhouts et al., 1991). This replicon has been exploited to construct various 
(a)

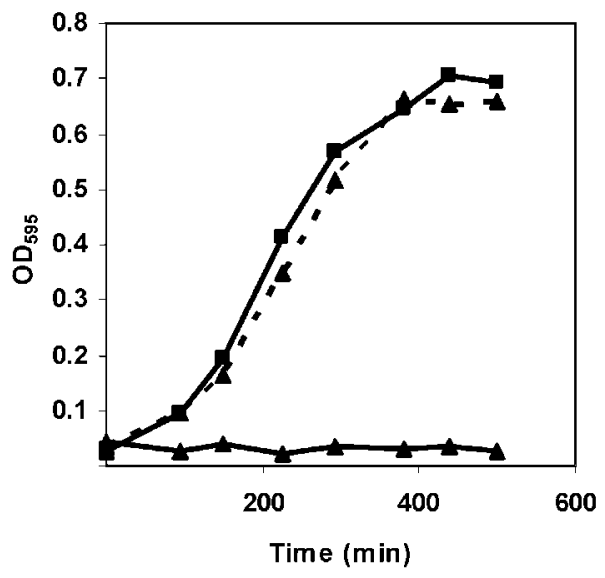

(b)

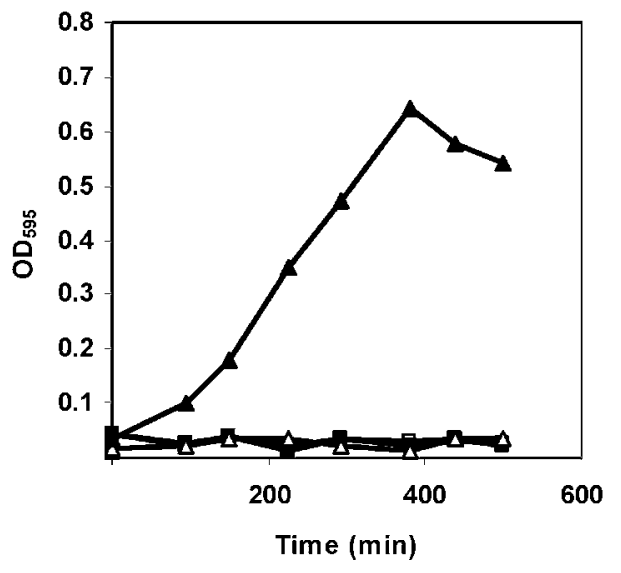

Fig. 2. Complementation of the $g \ln A$ deletion in S. pneumoniae D39nisRK. (a) Growth of strains D39nisRK (squares) and TK100 (D39nisRK $\Delta g / n A$; $\mathrm{Spec}^{\mathrm{R}}$ ) (triangles) in CDM containing $0.4 \mathrm{mg}$ glutamate $\mathrm{ml}^{-1}$ (solid lines), and growth of TK100 in CDM containing $0.4 \mathrm{mg}$ glutamine $\mathrm{ml}^{-1}$ (dashed line). (b) Growth of strain TK100 harbouring either plasmid pNG8048E (squares) or pTK6 (triangles) in CDM containing $0.4 \mathrm{mg}$ glutamate $\mathrm{ml}^{-1}$ and no glutamine. Nisin was added at a concentration of 0 (open symbols) or $2 \mathrm{ng} \mathrm{ml}^{-1}$ (black symbols).

molecular tools in L. lactis, and it functions in both Grampositive and Gram-negative bacteria (Kok et al., 1984). Replication of pORI vectors, which are derivatives of pWV01, is dependent on an in trans copy of the pWV01 replication initiation gene repA (Leenhouts et al., 1991).

To maintain the pORI vectors in S. pneumoniae, strain D39repA was constructed, which carries the repA gene, driven by a constitutive promoter, in the $b g a A$ locus (Fig. 3a). Like strain D39nisRK, D39repA does not display endogenous $\beta$-galactosidase activity (Fig. 3b). Subsequently, we tested whether the RepA-dependent plasmid pORI13 (Sanders et al., 1998), which contains a promoterless E. coli lacZ for transcriptional fusions preceded by a lactococcal ribosome-binding site, a multiple cloning site and stop codons in all three reading frames, could be employed as an integrative and replicative lac $Z$ reporter vector in $S$. pneumoniae strains D39nisRK and D39repA, respectively. To this end, the promoter of $\operatorname{cps} A$, the first gene of the locus, which encodes enzymes necessary for capsule production, was cloned upstream of the lac $Z$ of pORI13. The resulting construct was either integrated into the chromosome of strain D39nisRK or introduced into strain D39repA. The plasmid could be isolated from D39repA but not from D39nisRK (data not shown). Moreover, D39repA could be transformed easily with empty pORI13, while wild-type D39 could not, showing that pORI13 is maintained as an autonomous plasmid in D39repA. The cpsA promoter is active in GM17 medium, and lac $Z$ expression is approximately threefold higher in strain D39repA than in strain D39nisRK (Fig. 3b), indicating that pORI13 is present as a low-copy-number plasmid in the former, giving $\beta$-galactosidase activity close to that of a single-copy situation in the chromosome. Likewise, TIGR4 and R6 derivatives harbouring repA in the bgaA locus were able to replicate pORI13 (data not shown).

Thus, this system enables the use of the lac $Z$ expression plasmid pORI13 both for integration and as a low-copynumber plasmid, allowing detailed investigation of promoter function. Another advantage of having a RepA ${ }^{+}$ derivative of $S$. pneumoniae is that there is a range of different pORI vectors, with various antibiotic-resistance genes and extensive multiple-cloning sites (Leenhouts et al., 1996, 1998b), which can be maintained as replicative plasmids in D39repA as well (data not shown). In addition, pORI13 can be combined with the NICE system described above, since plasmid pNG8048E, containing the nisin-inducible promoter, provides the pWV01 repA gene required for the replication of pORI13 (data not shown).

\section{Construction of unmarked chromosomal mutations with pORI280 in S. pneumoniae}

Generally, mutant $S$. pneumoniae strains are constructed by substitution of a target gene with an antibiotic-resistance gene via a double cross-over event. This method has several disadvantages. First, it could cause polar effects on the expression of downstream genes, especially when the target gene is one of the first genes in an operon. Second, the introduction of point mutations or small in-frame deletions in a wild-type gene on the chromosome is not possible. Third, construction of strains containing multiple mutations could be hampered by the lack of sufficient antibiotic markers. The introduction of unmarked mutations into the chromosome would circumvent these drawbacks.

Previously, $r p s L$ has successfully been applied as a counterselectable marker in S. pneumoniae to construct unmarked mutations (Sung et al., 2001). However, this system requires the presence of a mutation in the wild-type chromosomal copy of $r p s L$. Furthermore, the mutated $r p s L$ allele spontaneously converts with low frequency to wild-type, giving rise to false positives (Sung et al., 2001). Unmarked mutants 
(a)

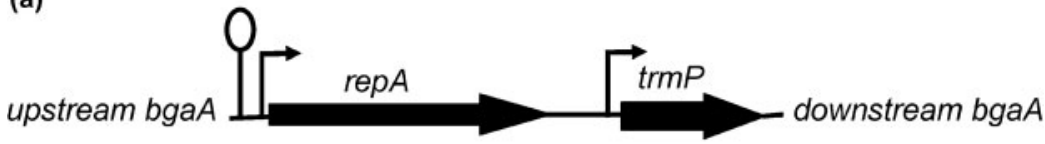

(b)

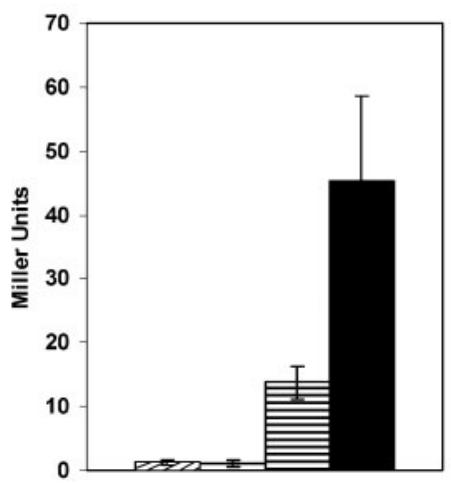

Fig. 3. Plasmid-based lac $Z$ expression system in $S$. pneumoniae. (a) Schematic representation of the $\operatorname{rep} A$ gene integrated in the chromosome at the bga $A$ locus of $S$. pneumoniae D39. The full-length $b g a A$ gene was removed from the chromosome. trmP, trimethoprim resistance marker; stem-loop indicates terminator structure (predicted $\Delta G^{0}$ $\left.-20.8 \mathrm{kcal} \mathrm{mol}^{-1}, \quad-87.0 \mathrm{~kJ} \mathrm{~mol}^{-1}\right)$; rightpointing arrows indicate promoters. (b) $\beta$ Galactosidase activity of D39nisRK (diagonal hatching), D39repA (white bar), D39nisRK carrying pJB2 (pORI13::PcpsA) integrated via single cross-over in the promoter region of cpsA (horizontal hatching), and D39repA harbouring pJB2 as a plasmid (black bar). Error bars show the standard deviation from three independent experiments. have also been constructed in S. pneumoniae by overlapextension PCR mutagenesis (Standish et al., 2005) and by direct transformation with a PCR fragment containing the desired mutation (Iannelli \& Pozzi, 2004). With these methods, selection for mutant clones is done by checking for antibiotic sensitivity and by colony PCR.

We investigated whether the lactococcal plasmid pORI280, which contains a constitutively expressed lac $Z$ and an erythromycin-resistance gene, can be used for the introduction of unmarked mutations in S. pneumoniae. pORI280 was originally developed to generate unmarked mutations in the chromosome of L. lactis independent of the genotype of the host, and it allows the screening of possible mutant clones on plates by visual blue/white selection (Leenhouts et al., 1996). We used gene spr0443 $(g \ln R)$ to test the system in $S$. pneumoniae. To this end, chromosomal fragments of $800 \mathrm{bp}$ surrounding $g \ln R$ were inserted into pORI280, and the construct (pTK7) was used to transform S. pneumoniae D39 with selection for erythromycin resistance (see Fig. 4 for a schematic representation of the procedure). As replication of pORI280 is dependent on RepA, the transformation leads to single cross-over integration of the construct into the chromosome. After growing several erythromycin-resistant, LacZ-positive integrants as separate cultures for 30-50 generations (culturing two to four times until stationary

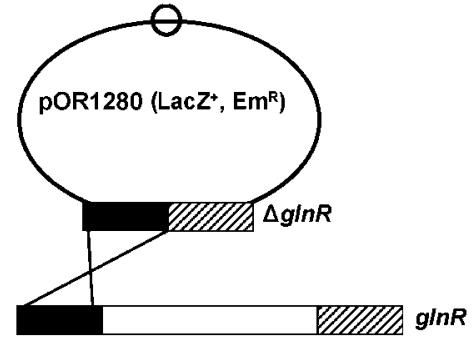

(1)

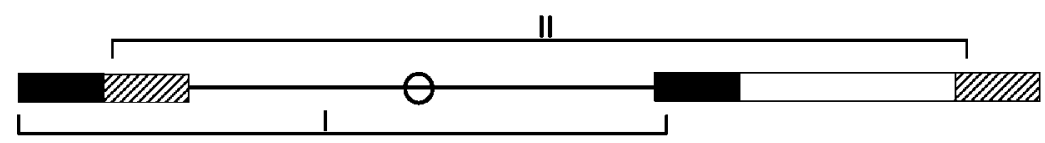

(2) I

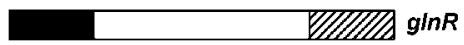

II
Fig. 4. Schematic representation of the use of pORI280 for the construction of unmarked deletions in the chromosome of $S$. pneumoniae. pORI280 containing the left (black rectangle) and right (hatched rectangle) flanking regions of $g \ln R$ is integrated into the chromosome via single cross-over (1); small circle, origin of replication of pWV01. For simplicity, only integration via one side is depicted. LacZ ${ }^{+}$, erythromycin-resistant integrants are grown without selection to allow the excision of pORI280 in a second recombination event through either I or II (2). This results in white, erythromycin-sensitive clones that have either reverted to the wild-type (I) or contain the deleted $g \ln R$ (II). 
phase) without antibiotic selection, cells were plated on $\mathrm{X}$-Gal medium to screen for clones that had lost the plasmid as the result of a second recombination event. Of the colonies, $0.5 \%$ were both white and erythromycin sensitive, indicating excision of the plasmid from the chromosome. Of these white erythromycin-sensitive colonies, $80 \%$ contained the desired mutation, as verified by PCR, Southern blotting and nucleotide sequencing (data not shown). In the same way, we successfully introduced two point mutations in the chromosomal copy of $g \ln R$ of S. pneumoniae D39. Both mutant strains will be described in detail elsewhere. An advantage of the use of pORI280 compared to the methods mentioned above is that possible mutants can be easily selected on the basis of their white colour on plates with $\mathrm{X}-\mathrm{Gal}$. Thus, this system provides an efficient way to obtain unlabelled mutants in S. pneumoniae.

\section{Conclusions}

In this paper, we report the successful development and employment of important molecular tools for the study of gene function and gene expression in S. pneumoniae. These tools were adapted from the L. lactis toolbox and complement existing genetic methods. A general benefit is that all cloning steps with $S$. pneumoniae DNA can be performed in $L$. lactis, which in our hands gives fewer problems than $E$. coli. Taken together, the tools offer new possibilities for molecular research on S. pneumoniae and will help to unravel the molecular mechanisms that underlie infection by and the pathogenesis of this important human pathogen.

\section{ACKNOWLEDGEMENTS}

T. G. K. and J. J. E. B. are supported by IOP grant IGE03002. We thank K. Thiadens and A. de Vos van Steenwijk for excellent technical assistance. We thank D. Morrison for the generous gift of competencestimulating peptide. We thank P. Burghout, H. Bootsma, W. Hendriksen and P. Hermans for stimulating discussions. We also thank P. Hermans for providing plasmid pKOT and strains D39, R6 and TIGR4.

\section{REFERENCES}

Acebo, P., Nieto, C., Corrales, M. A., Espinosa, M. \& Lopez, P. (2000). Quantitative detection of Streptococcus pneumoniae cells harbouring single or multiple copies of the gene encoding the green fluorescent protein. Microbiology 146, 1267-1273.

Adams, M. H. \& Roe, A. S. (1945). A partially defined medium for cultivation of Pneumococcus. J Bacteriol 49, 401-409.

Avery, O. T., Macleod, C. M. \& McCarty, M. (1944). Studies on the chemical nature of the substance inducing transformation of pneumococcal types. Induction of transformation by a desoxyribonucleic acid fraction isolated from Pneumococcus type III. Mol Med 1, 137-158.

Bartilson, M., Marra, A., Christine, J., Asundi, J. S., Schneider, W. P. \& Hromockyj, A. E. (2001). Differential fluorescence induction reveals Streptococcus pneumoniae loci regulated by competence stimulatory peptide. Mol Microbiol 39, 126-135.
Bogaert, D., de Groot, R. \& Hermans, P. W. (2004). Streptococcus pneumoniae colonisation: the key to pneumococcal disease. Lancet Infect Dis 4, 144-154.

Chan, P. F., O'Dwyer, K. M., Palmer, L. M. \& 8 other authors (2003). Characterization of a novel fucose-regulated promoter (PfcsK) suitable for gene essentiality and antibacterial mode-of-action studies in Streptococcus pneumoniae. J Bacteriol 185, 2051-2058.

Chomczynski, P. \& Qasba, P. K. (1984). Alkaline transfer of DNA to plastic membrane. Biochem Biophys Res Commun 122, 340-344.

Claverys, J. P., Dintilhac, A., Pestova, E. V., Martin, B. \& Morrison, D. A. (1995). Construction and evaluation of new drug-resistance cassettes for gene disruption mutagenesis in Streptococcus pneumoniae, using an ami test platform. Gene 164, 123-128.

de Ruyter, P. G., Kuipers, O. P., Beerthuyzen, M. M., van AlenBoerrigter, I. \& de Vos, W. M. (1996a). Functional analysis of promoters in the nisin gene cluster of Lactococcus lactis. J Bacteriol 178, 3434-3439.

de Ruyter, P. G., Kuipers, O. P. \& de Vos, W. M. (1996b). Controlled gene expression systems for Lactococcus lactis with the food-grade inducer nisin. Appl Environ Microbiol 62, 3662-3667.

Eichenbaum, Z., Federle, M. J., Marra, D., de Vos, W. M., Kuipers, O. P., Kleerebezem, M. \& Scott, J. R. (1998). Use of the lactococcal nisA promoter to regulate gene expression in Gram-positive bacteria: comparison of induction level and promoter strength. Appl Environ Microbiol 64, 2763-2769.

Fisher, S. H. \& Sonenshein, A. L. (1984). Bacillus subtilis glutamine synthetase mutants pleiotropically altered in glucose catabolite repression. J Bacteriol 157, 612-621.

Hancock, R. E. (2005). Mechanisms of action of newer antibiotics for Gram-positive pathogens. Lancet Infect Dis 5, 209-218.

Holo, H. \& Nes, I. F. (1995). Transformation of Lactococcus by electroporation. Methods Mol Biol 47, 195-199.

Hoskins, J., Alborn, W. E., Jr, Arnold, J. \& 40 other authors (2001). Genome of the bacterium Streptococcus pneumoniae strain R6. $J$ Bacteriol 183, 5709-5717.

lannelli, F. \& Pozzi, G. (2004). Method for introducing specific and unmarked mutations into the chromosome of Streptococcus pneumoniae. Mol Biotechnol 26, 81-86.

Israelsen, H., Madsen, S. M., Vrang, A., Hansen, E. B. \& Johansen, E. (1995). Cloning and partial characterization of regulated promoters from Lactococcus lactis Tn917-lacZ integrants with the new promoter probe vector, pAK80. Appl Environ Microbiol 61, 2540-2547.

Johansen, E. \& Kibenich, A. (1992). Isolation and characterization of IS1165, an insertion sequence of Leuconostoc mesenteroides subsp. cremoris and other lactic acid bacteria. Plasmid 27, 200-206.

Kleerebezem, M., Beerthuyzen, M. M., Vaughan, E. E., de Vos, W. M. \& Kuipers, O. P. (1997). Controlled gene expression systems for lactic acid bacteria: transferable nisin-inducible expression cassettes for Lactococcus, Leuconostoc, and Lactobacillus spp. Appl Environ Microbiol 63, 4581-4584.

Kok, J., van der Vossen, J. M. \& Venema, G. (1984). Construction of plasmid cloning vectors for lactic streptococci which also replicate in Bacillus subtilis and Escherichia coli. Appl Environ Microbiol 48, 726-731.

Kramer, N. E. (2005). Nisin-resistance in Gram-positive bacteria. $\mathrm{PhD}$ thesis, University of Groningen, The Netherlands.

Kuipers, O. P., Beerthuyzen, M. M., de Ruyter, P. G., Luesink, E. J. \& de Vos, W. M. (1995). Autoregulation of nisin biosynthesis in Lactococcus lactis by signal transduction. J Biol Chem 270, 27299-27304.

Kuipers, O. P., Ruyter, P. G., Kleerebezem, M. \& Vos, W. M. (1998). Quorum sensing controlled gene expression in lactic acid bacteria. J Biotechnol 64, 15-21. 
Lee, M. S., Seok, C. \& Morrison, D. A. (1998). Insertion-duplication mutagenesis in Streptococcus pneumoniae: targeting fragment length is a critical parameter in use as a random insertion tool. Appl Environ Microbiol 64, 4796-4802.

Leenhouts, K. J., Kok, J. \& Venema, G. (1991). Lactococcal plasmid pWV01 as an integration vector for lactococci. Appl Environ Microbiol 57, 2562-2567.

Leenhouts, K., Buist, G., Bolhuis, A., ten Berge, A., Kiel, J., Mierau, I., Dabrowska, M., Venema, G. \& Kok, J. (1996). A general system for generating unlabelled gene replacements in bacterial chromosomes. Mol Gen Genet 253, 217-224.

Leenhouts, K., Bolhuis, A., Venema, G. \& Kok, J. (1998a). Construction of a food-grade multiple-copy integration system for Lactococcus lactis. Appl Microbiol Biotechnol 49, 417-423.

Leenhouts, K., Venema, G. \& Kok, J. (1998b). A lactococcal pWV01 based integration toolbox for bacteria. Methods Cell Sci 20, 35-50.

Luo, P., Li, H. \& Morrison, D. A. (2004). Identification of ComW as a new component in the regulation of genetic transformation in Streptococcus pneumoniae. Mol Microbiol 54, 172-183.

Marra, A., Asundi, J., Bartilson, M. \& 7 other authors (2002). Differential fluorescence induction analysis of Streptococcus pneumoniae identifies genes involved in pathogenesis. Infect Immun 70, $1422-1433$.

Mierau, I. \& Kleerebezem, M. (2005). 10 years of the nisincontrolled gene expression system (NICE) in Lactococcus lactis. Appl Microbiol Biotechnol 68, 705-717.

Ng, W. L., Kazmierczak, K. M. \& Winkler, M. E. (2004). Defective cell wall synthesis in Streptococcus pneumoniae R6 depleted for the essential PcsB putative murein hydrolase or the VicR (YycF) response regulator. Mol Microbiol 53, 1161-1175.

Pestova, E. V. \& Morrison, D. A. (1998). Isolation and characterization of three Streptococcus pneumoniae transformation-specific loci by use of a lac $Z$ reporter insertion vector. J Bacteriol 180, 2701-2710.
Poolman, B. \& Konings, W. N. (1988). Relation of growth of Streptococcus lactis and Streptococcus cremoris to amino acid transport. J Bacteriol 170, 700-707.

Pozzi, G., Masala, L., lannelli, F., Manganelli, R., Havarstein, L. S., Piccoli, L., Simon, D. \& Morrison, D. A. (1996). Competence for genetic transformation in encapsulated strains of Streptococcus pneumoniae: two allelic variants of the peptide pheromone. J Bacteriol 178, 6087-6090.

Rollema, H. S., Kuipers, O. P., Both, P., de Vos, W. M. \& Siezen, R. J. (1995). Improvement of solubility and stability of the antimicrobial peptide nisin by protein engineering. Appl Environ Microbiol 61, 2873-2878.

Sanders, J. W., Venema, G., Kok, J. \& Leenhouts, K. (1998). Identification of a sodium chloride-regulated promoter in Lactcoccus lactis by single-copy chromosomal fusion with a reporter gene. Mol Gen Genet 257, 681-685.

Standish, A. J., Stroeher, U. H. \& Paton, J. C. (2005). The twocomponent signal transduction system RR06/HK06 regulates expression of cbpA in Streptococcus pneumoniae. Proc Natl Acad Sci U S A 102, 7701-7706.

Sung, C. K., Li, H., Claverys, J. P. \& Morrison, D. A. (2001). An rpsL cassette, janus, for gene replacement through negative selection in Streptococcus pneumoniae. Appl Environ Microbiol 67, 5190-5196.

Terzaghi, B. E. \& Sandine, W. E. (1975). Improved medium for lactic streptococci and their bacteriophages. Appl Environ Microbiol 29, 807-813.

Tettelin, H., Nelson, K. E., Paulsen, I. T. \& 36 other authors (2001). Complete genome sequence of a virulent isolate of Streptococcus pneumoniae. Science 293, 498-506.

van de Rijn, I. \& Kessler, R. E. (1980). Growth characteristics of group A streptococci in a new chemically defined medium. Infect Immun 27, 444-448.

Willett, N. P. \& Morse, G. E. (1966). Long-chain fatty acid inhibition of growth of Streptococcus agalactiae in a chemically defined medium. J Bacteriol 91, 2245-2250. 